\title{
Spontaneous intraperitoneal rupture of the bladder in association with transitional cell carcinoma
}

\author{
J.S. Budd
}

Department of Surgery, Princess Margaret Hospital, Okus Road, Swindon, SN1 4JU, Wilts, UK.

\begin{abstract}
Summary: A 79 year old woman presented with generalized peritonitis and was subsequently found to have a perforated bladder secondary to a tumour. This is a rare occurrence and a difficult diagnosis to make preoperatively.
\end{abstract}

\section{Introduction}

Perforation of the urinary bladder without a history of antecedent trauma is a rare clinical occurrence. In two reviews of the subject by Stone $^{1}$ and Bastable et al. ${ }^{2}$ totalling over 100 cases, it becomes apparent that perforation through a carcinoma is a very rare cause of spontaneous rupture. Most cases in the reviews, and other case reports, occurred in males and this is probably a reflection on the anatomical features of the male urethra making the male bladder more liable to distension and consequent perforation. The clinical presentation in atraumatic bladder perforation is usually as acute generalized peritonitis and the diagnosis is only rarely considered and is usually made at laparotomy. The following case of spontaneous rupture of the bladder presented in just this way and is unusual in that the perforation was in a female bladder and occurred through an area of transitional cell carcinoma.

\section{Case report}

A 79 year old female was admitted with a 12 hour history of generalized abdominal pain and incontinence of urine. She had complained of incontinence for many years but this had been considerably more troublesome just before admission. She also complained of dysuria and on questioning admitted to some haematuria, but only during the 24 hours prior to admission. She also gave a long history of intermittent epigastric pains. On examination she looked ill, was pyrexial and had signs of generalized peritonitis. Her white cell

Correspondence: J.S. Budd F.R.C.S.

Accepted: 9 September 1987 count was raised at $20 \times 10^{9} / 1$, electrolytes and amylase were normal but she did have a slightly raised urea of $10 \mathrm{mmol} / \mathrm{l}$. Although plain X-rays showed no evidence of free peritoneal gas a provisional diagnosis of a perforated peptic ulcer was made and a laparotomy carried out.

At laparotomy the peritoneal cavity was found to be filled with about 3 litres of foul smelling urine. A $1 \mathrm{~cm}$ perforation was identified in the vault of the bladder, to the right of the mid-line, and was centred in a large tumorous mass which occupied the right half of the bladder. A biopsy was taken and the perforation closed with catgut. Peritoneal lavage was performed, a drain was inserted into the abdominal cavity and the bladder was catheterized. Histology of the lesion showed a poorly differentiated invasive transitional cell carcinoma. The patient recovered well and subsequently underwent a 5 day course of palliative radiotherapy to the bladder tumour. She remained well for some months but has since died of metastatic disease.

\section{Discussion}

Spontaneous, non-traumatic rupture of the bladder is a rare occurrence and is associated with a high mortality. In association with neoplastic disease of the bladder it is very rare and most cases reported are in males. Cases of rupture through an area of squamous cell carcinoma in the female bladder have been previously reported, ${ }^{3,4}$ but so far as can be ascertained from the literature only one previous case of spontaneous rupture through a transitional cell carcinoma in a female can be found. ${ }^{3}$ Even in this case marked squamous metaplasia was reported in the initial biopsy specimen and histology of the tumour subsequently excised identified

(C) The Fellowship of Postgraduate Medicine, 1988 
a squamous cell carcinoma. This would therefore appear to be the first reported case of spontaneous

\section{References}

1. Stone, E. Spontaneous bladder rupture. Arch Surg 1931, 23: 129.

2. Bastable, J.R.G., De Jode, L.R. \& Warren, R.P. Spontaneous rupture of the bladder. Br J Urol 1959, 31: 78-86.

3. Jenkinson, L.R. Spontaneous intraperitoneal rupture of urinary bladder. Postgrad Med J 1981, 57: 269-270. rupture of the female bladder associated with a truly transitional cell carcinoma.

4. Huffman, J.L., Schraut, W. \& Bagley, D.M. Atraumatic perforation of bladder. Necessary differential in evaluation of acute condition of abdomen. Urology 1983, 22: 30-35.

5. Glasman, R.W. Perforation as a complication of carcinoma of the bladder. Br J Urol 1967, 39: 178-180. 